\title{
Spatially located visual CS effects in conditioned avoidance shuttle response acquisition in goldfish: Conditioned aversion or phototaxis?
}

\author{
D. J. ZERBOLIO, JR. and L. L. WICKSTRA \\ University of Missouri, St. Louis, Missouri 63121
}

\begin{abstract}
Goldfish (Carassius auratus) were trained in a conditioned avoidance shuttle response (CASR) situation, where the CS was a color change which occurred on the same, opposite, or both ends of the shuttle tank. One hundred acquisition trials were administered either in a single day or in 20 trials a day for 5 consecutive days. Earlier work, using a change of illumination level CS, found different CASR acquisition rates with 1 day of training vs. training over several days. The present study finds basically the same order of acquisition rates within a single day as over days, with the exception of one group. Differences between CS location acquisition rates in earlier work (with illumination level change) and those of the present study (color change without illumination level change) are explained on the basis of negative phototaxis or confusion reactions occurring under the illumination level change situation.
\end{abstract}

Earlier Zerbolio and Wickstra (1976) confirmed Gallon's (1974) finding that goldfish (Carassius auratus) acquire a conditioned avoidance shuttle response (CASR) as a function of CS location, where, in a single training session of 100 trials, the order of maximum to minimum CASR performance was same, both, and opposite. But Wickstra and Zerbolio (1976), with training over days instead of in a single day, found the CASR order to be both maximum, same intermediate, and opposite minimum. These latter results are consistant with Kish's (1955) finding that avoidance acquisition is a function of the CS change magnitude. To account for the difference in order between the single-day paradigm (same, both, opposite) and the multiday paradigm (both, same, opposite), Wickstra and Zerbolio (1976) postulated that the sudden increase in illumination for the CS onset condition produces a negative phototactic reaction in the single-day paradigm, but that this reaction acquires cue characteristics in the multiday paradigm. According to this position, the negative phototactic reaction augments performance in the CS onset same condition both for training in a single day and over days, and thus does not produce differential effects. However, the negative phototaxis elicited by light onset has different behavioral effects in the CS onset opposite and CS onset both single-day paradigms. For the CS onset opposite single-day condition, the subject is required to approach a stimulus eliciting negative phototaxis which markedly debilitates CASR acquisition. For the CS onset both single-day condition, the subject is required to approach the negative phototactic stimulus, but is also leaving a negative phototactic stimulus. Thus, CASR acquisition performance is augmented over that of the CS onset opposite (approaching the stimulus only). Over days, the negative phototactic reaction is hypothesized to acquire response cue characteristics which augment CASR acquisition performance.
For the CS offset situation, the sudden decrease in illumination was hypothesized to be confusing to the subject (Gallon, 1974; Wickstra \& Zerbolio, 1976), but the confusion reaction also acquires cue properties which augment avoidance performance over days.

If the effect of the differential reactions due to the hypothesized negative phototaxis or confusion reaction is reasonable, the CASR acquisition rates ought to be comparable for the single-day paradigm and multiday paradigm if the experimental conditions which produce them can be eliminated. The present study attempts to accomplish this by using a constant illumination level for the ITI and CS trial periods, but changing the color of illumination differentially during the CS period. A color change CS can be as easily localized as an illumination change, and differential color detection is within the sensory capabilities of goldfish (Yager, 1968).

\section{METHOD}

\section{Subjects}

Seventy-two goldfish, $5-8 \mathrm{~cm}$ long, acquired from Ozark Fisheries, were used. All subjects were housed in 30-gal aquaria for at least $48 \mathrm{~h}$ prior to running. Forty-eight hours prior to running, the subjects were transferred to individual $7.5 \times 11.5 \times$ $12.5 \mathrm{~cm}$ deep tanks. Temperature $\left(21^{\circ} \mathrm{C}\right)$ and $\mathrm{pH}(7 \pm .1)$ were held constant throughout. All housing conditions were well aerated and filtered and subjects were fed daily.

\footnotetext{
Apparatus

All subjects were run in two identical $29.2 \times 11.4 \times 11.4 \mathrm{~cm}$ deep shuttle tanks, separated by a center hurdle $6.35 \mathrm{~cm}$ high. Water clearance of $2.5 \mathrm{~cm}$ over the top of the hurdle was maintained. Photo cells located at the ends of the hurdle monitored all shuttling activity. A blue and green 7-W 110 ac lamp was located at each tank end. Both lamps were independently programmable. Diffusing plates were affixed between each tank end and the lamps. The US was delivered via $28 \times 10.2 \mathrm{~cm} 22 \mathrm{gg}$ stainless steel plates affixed to the internal sides of each tank. The US was generated by variable isolated transformers, individually metered and monitored at $7.5 \mathrm{~V}$ ac.
} 
Procedure

All subjects were given 100 acquisition trials, half $(n=36)$ in a single session $(100 / 1)$ and the remainder $(n=36)$ in sessions of 20 trials for 5 consecutive days (20/5). Each of these groups (100/1 and 20/5) were further divided into three CS location conditions of CS same, CS opposite, and CS both ( $n=12$ each). Additionally, each location group had half $(n=6)$ run with a blue ITI/green CS and the remainder $(n=6)$ with a green ITI/blue CS stimulus change to balance any color effects. Thus, for example, the blue ITI/green CS condition had both blue lamps (both ends) on during the ITI. The trial CS color change involved changing the appropriate lamps. For the CS same location, the green lamp was lit and the blue light extinguished on the same end as that occupied by the subject. For the CS opposite location, the green lamp was lit and the blue extinguished on the side opposite the subject. For the CS both location, the change occurred on both ends of the tank. Approximately equal levels of illumination were present in the tanks at all times. A trial consisted of an appropriate CS change period for $10 \mathrm{sec}$, followed by a 2.2-sec US period when a maximum of five 200-msec-on/300-msec-off 7.5 ac shocks could be delivered. The ITI was a VI $60 \mathrm{sec}$. Any initial shuttle response occurring in the 10 -sec CS-US period was recorded as an avoidance, and any initial shuttle response during the US period was recorded as an escape. The first response from CS onset to US offset period was recorded appropriately and immediately terminated further stimulation for that trial. All events were automatically programmed and responses were recorded both on counters and paper tape by appropriate circuitry.

\section{RESULTS}

A 2 by 3 by 2 by 5 repeated ANOVA for training conditions $(100 / 1$ vs. $20 / 5)$ by CS location (same, opposite or both) by ITI/CS conditions (blue ITI/green CS or green ITI/blue CS) by blocks of 20 trials on avoidance responses was calculated. Significant effects for Training blocks $\left[\mathrm{F}(4,240)=46.76, \mathrm{p}<.01, \omega^{2}=.200\right]$ and a significant interaction for Training Conditions $(100 / 1$ vs. $20 / 5)$ by CS Location $[F(2,60)=5.08$, $\left.\mathrm{p}<.01, \omega^{2}=.040\right]$ were found. All other results were trivial and accounted for much smaller amounts of variance (Hays, 1963; Kirk, 1968; Vaughn \& Corballis, 1969; Zerbolio, 1973). These results appear in Figure 1.

Additional comparisons of overall avoidance found no differences among the 100/1 same and both and 20/5 same, opposite, and both conditions, but all groups were superior to the $100 / 1$ opposite group $[\operatorname{HSD}(6,60)$ $=10.23$; differences are 26.7, 21.0,27.0, 25.2, and 20.4, respectively] (Winer, 1971).

Analyses of variance for the ITI shuttle rates for the $100 / 1$ and 20/5 groups were calculated separately. For the $100 / 1$ groups, no significant differences were found. For the $20 / 5$ groups, reliable differences for CS location were found $[F(2,30)=3.465, p<.05]$. These results are also shown in Figure 1. No effects for the blue ITI/green CS vs. green ITI/blue CS balancing was detected, either in the avoidance response or the ITI shuttle rate data.

\section{DISCUSSION}

The present data show that CASR acquisition rates are equivalent for CS same and CS both conditions, for both the singleday $(100 / 1)$ and multiday $(20 / 5)$ training procedures. It appears that when the negative phototaxic and confusion reactions are absent, the important variable is magnitude of CS change on the side occupied by the subject at trial onset. The CASR, then, is unaffected by additional reactions such as those elicited by a sudden decrease in illumination (confusion reaction) or a sudden increase in illumination (negative phototaxis). The acquisition rate of the CS opposite single-day group also fits, since, for the subject, this condition presents the least magnitude of CS change. But the CASR rate of the CS opposite multiday group is surprising, as its acquisition rate is comparable to all other groups except the CS opposite single-day group. Further analysis of these data suggest an explanation. Both CS opposite groups are at about the same level following the first block of 20 trials. Following that, the CS opposite multiday group shows a very large (comparatively) increase in CASR performance, and this is accompanied by a large increase in shuttle rate activity.

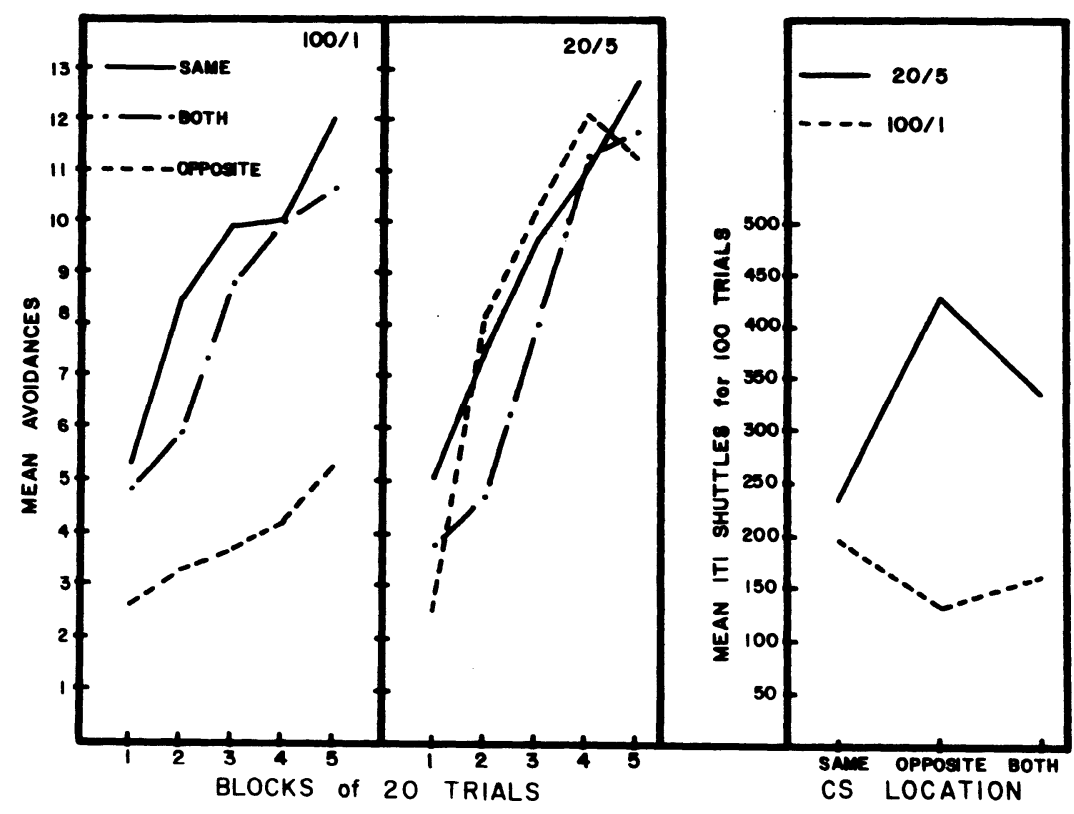

Figure 1. The mean avoidances by blocks of 20 trials and mean ITI for 100 trials for training in a single day $(100 / 1)$ and over 5 days $(20 / 5)$ and for each of three CS locations: CS same, CS opposite, and CS both. 
This increase in shuttle rate activity shows up in the analysis, in that this group has a significantly higher overall ITI response rate than the other groups. It is possible that, given the minimal CS change, subjects in this condition acquire a high nonspecific generalized activity rate rather than a stimulus-specific avoidance response. The opportunity to acquire this high rate may be limited to a multiday paradigm. This is consistent with earlier findings by Zerbolio and Wickstra (1975) which indicate that, on the first day of training, one of the effects of shock is to markedly reduce overall activity rate.

In sum, then, the present data strongly suggest that earlier work by Gallon (1974) and Zerbolio and Wickstra (1976), investigating the effect of CS location on CASR acquisition rates, may have confounded acquisition rate with reactions to sudden increases or decreases in localized illumination level. Thus, Gallon's (1974) conclusion of the acquisition of a "conditioned aversion" to the CS (light onset) may well have been simply the subject responding in the negative phototactic manner. A similar explanation could hold true for the hypothesized "confusion reaction" elicited by sudden light offset. The present data strongly support such a conclusion.

\section{REFERENCES}

Gallon, R. L. Spatial location of a visual signal and shuttle box avoidance acquisition by goldfish (Carassius auratus). Journal of Comparative and Physiological Psychology, 1974, 86, 316-321.

Hays, W. L. Statistics for psychologists. New York: Holt, Rinehart, and Winston, 1963.
KIRK, R. E. Experimental design: Procedures for the behavioral sciences. Belmont, CA: Brooks/Cole, 1968.

Kish, G. B. Avoidance learning to the onset and cessation of conditioned stimulus energy. Journal of Experimental Psycho$\log y, 1955,50,31-38$.

Vaughn, B. M., \& Corballis, M. C. Beyond tests of significance: Estimating strength of effects in selected ANOVA designs. Psychological Bulletin, 1969, 72, 204-213.

Wickstra, L. L., \& Zerbolio, D. J., JR. Spatially located visual CS effects on conditioned avoidance shuttle response (CASR) acquisition in goldfish (Carassius auratus): Training over days. Bulletin of the Psychonomic Society, 1976, 8, 124-126.

WINER, B. J. Statistical principles in experimental design (2nd ed.). New York: McGraw-Hill, 1971.

YAGER, D. Behavioral analysis of color sensitivities in goldfish. In D. Ingle (Ed.), The central nervous system and fish behavior. Chicago: University of Chicago Press, 1968.

Zerbolio, D. J. Temperature-dependent learning in goldfish: A multi-trial active avoidance situation. Behavioral Biology, $1973,8,775-761$.

Zerbolio, D. J., JR., \& Wickstra, L. L. The effect of power (US intensity times US duration) on shuttlebox avoidance acquisition in goldfish. Bulletin of the Psychonomic Society, $1975,4,345-347$.

ZERBolio, D. J., JR., \& Wickstra, L. L. Spatially located visual CS effects on conditioned shuttlebox avoidance in goldfish (Carassius auratus): Further analysis. Bulletin of the Psychonomic Society, 1976, 7, 503-505.

(Received for publication May 3, 1976.) 